\title{
Cloud cover analysis with METEOSAT-5 during INDOEX
}

\author{
Geneviève Sèze \\ Laboratoire de Météorologie Dynamique du CNRS, Paris, France
}

Hanna Pawlowska

Institute of Geophysics, Warsaw University, Warsaw, Poland

\begin{abstract}
During the Indian Ocean Experiment (INDOEX), METEOSAT-5 positioned at $63^{\circ} \mathrm{E}$ provided observation of the visible and infrared radiance field over the Indian Ocean. A cloud classification process using the dynamic cluster method is applied to these data. For the 3 months of the experiment (January-March 1999), daily maps of the cloud cover type are built for 0730 and 0900 UTC. The occurrence frequency of clear sky, lowand high-level cloud cover is examined. These frequencies are compared to the International Satellite Cloud Climatology Program (ISCCP) D1 data set for the period 1984 to 1994. The Indian Ocean region can be classified in three zones. In the north subtropics, clear sky and small cumulus occur at least $90 \%$ of the time. Near the coast of India, clear sky is as frequent as 80 to $100 \%$. The Intertropical Convergence Zone,

characterized by the occurrence frequency of high-level clouds greater than $30 \%$, spreads from Indonesia to North Madagascar. Near Indonesia, high-level cloud cover occurs more than $55 \%$ of the time. In the south subtropics, low cloud cover is the most frequent. In the eastern part the occurrence frequency reaches $80 \%$. This percentage decreases along the western side of the ocean where low clouds break up. Between the African coast and Madagascar, high-level clouds are frequent. The mean spatial features found are in agreement with the ISCCP climatology, except for the eastern part of the south subtropics. A regional comparison shows the difficulty of making the analysis of interannual variations of cloud cover obtained from various cloud cover retrievals applied to different satellite data sets. This difficulty arises from the nonneglectable percentage of satellite pixels which can contain some very small low clouds.
\end{abstract}

\section{Introduction}

Climatologies of the cloud cover over the Indian Ocean area were very few owing to the lack of easily accessible geostationary satellite data. In June 1998 upon request from the scientists involved in the Indian Ocean Experiment (INDOEX) $[R a-$ manathan et al., 1996], the European Organization for the Exploitation of Meteorological Satellites (EUMETSAT) shifted the METEOSAT -5 to $63^{\circ} \mathrm{E}$, thus offering a unique data set for the study of spatial and temporal variability of the cloud cover over the Indian Ocean region. In preparation for the intensive phase of the experiment, several studies of the cloud cover have been made. Using the Indian geostationary satellite (INSAT) data, the International Satellite Cloud Climatology Program (ISCCP) data, and surface observations [Bony et al., 2000], the occurrence of nonoverlapped low clouds over the Indian Ocean during the winter monsoon was examined. Roca and Ramanathan [2000], using the Indian geostationary satellite (INSAT) data, document the characteristics of the deep convective systems over the Indian Ocean.

In this paper, the occurrence of the main cloud type cover over the Indian Ocean during the 3-month period (JanuaryMarch 1999) of the INDOEX experiment is examined. Maps of cloud type occurrence frequency are built. The spatial features of these maps, which are related to the main climate Copyright 2001 by the American Geophysical Union.

Paper number 2001JD900097.

0148-0227/01/2001JD900097\$09.00 regimes present in the region, are examined and compared with whose which can be observed during the 1984 to 1994 period with the ISCCP D1 data. A regional analysis is performed. The coherence between the results found for 1999 in our study and for the 1984 to 1994 period with the ISCCP D1 data set is studied.

During the comprehensive field experiment from February to March 1999 the operational management was highly dependent on the METEOSAT images. An operational cloud analysis procedure was set up specifically to detect low clouds whose life cycle and optical properties are suspected to be affected by the presence of tropospheric aerosols [Twomey, 1974; Ackerman et al., 2000]. This operational procedure, based on a threshold technique, used in particular the water vapor channel at $6.7 \mu \mathrm{m}$ to separate low clouds from cirrus. It performed very well in this task, as evaluated by the field scientists and confirmed by the flight observations. An updated version of this procedure is applied by Roca et al. [this issue] to study convection and water vapor distributions during INDOEX.

In this paper, another approach is presented. Cloud classification developed by Sèze and Desbois [1987] and Raffaelli and Sèze [1995] is applied to the visible and infrared METEOSAT-5 data. Thanks to the use of the local spatial variability of the visible and infrared radiances, this procedure, a clustering technique, allows a fairly good separation of completely clear-sky pixels from those partially covered by broken low clouds or thin cirrus and a detailed classification of cloud types [Sèze et al., 1999]. For the 3-month period, maps of the 


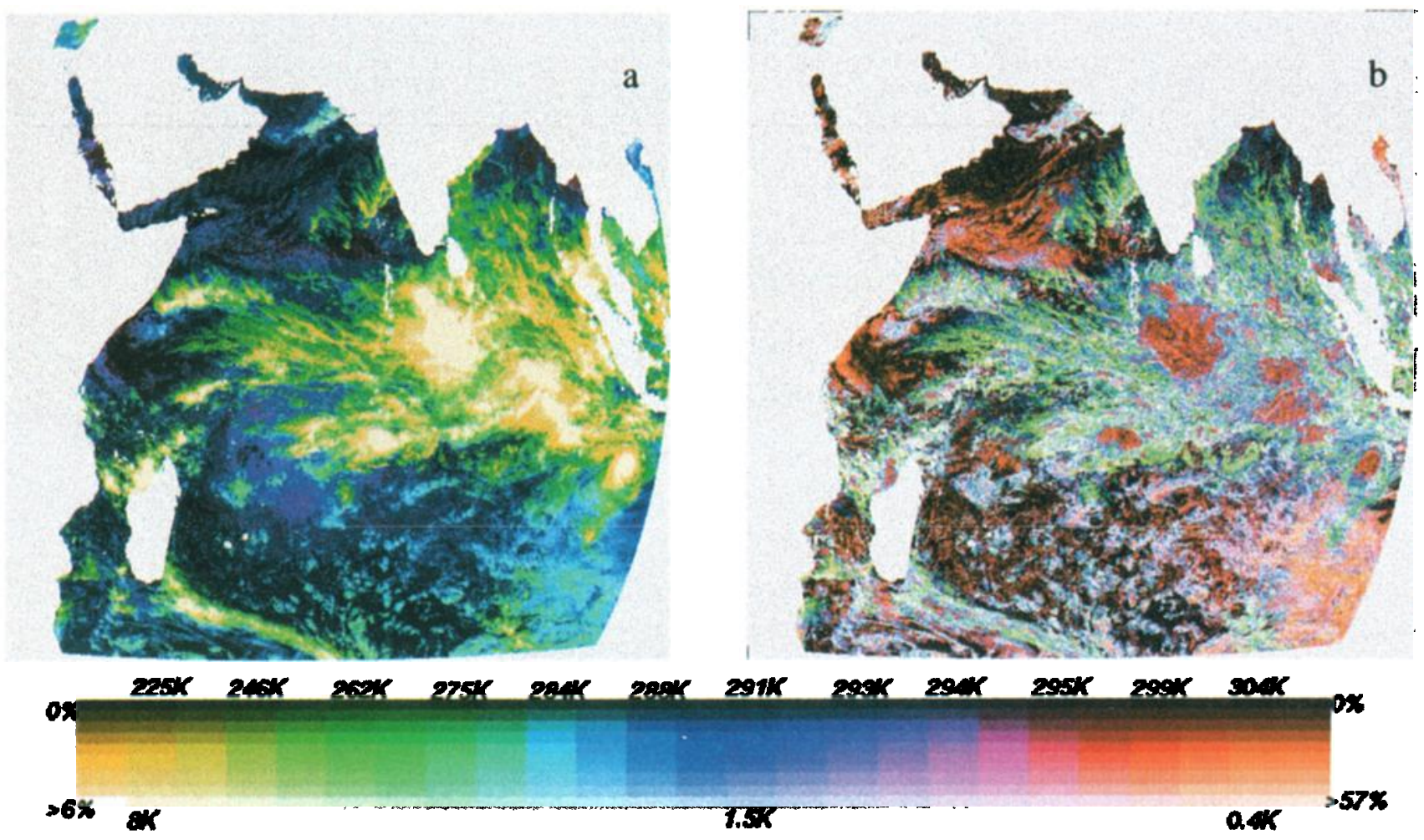

Plate 1. (a) Composite image of reflectance and brightness temperature field. (b) Composite image of the local standard deviation of the brightness temperature and reflectance. For details of legend description, see text.

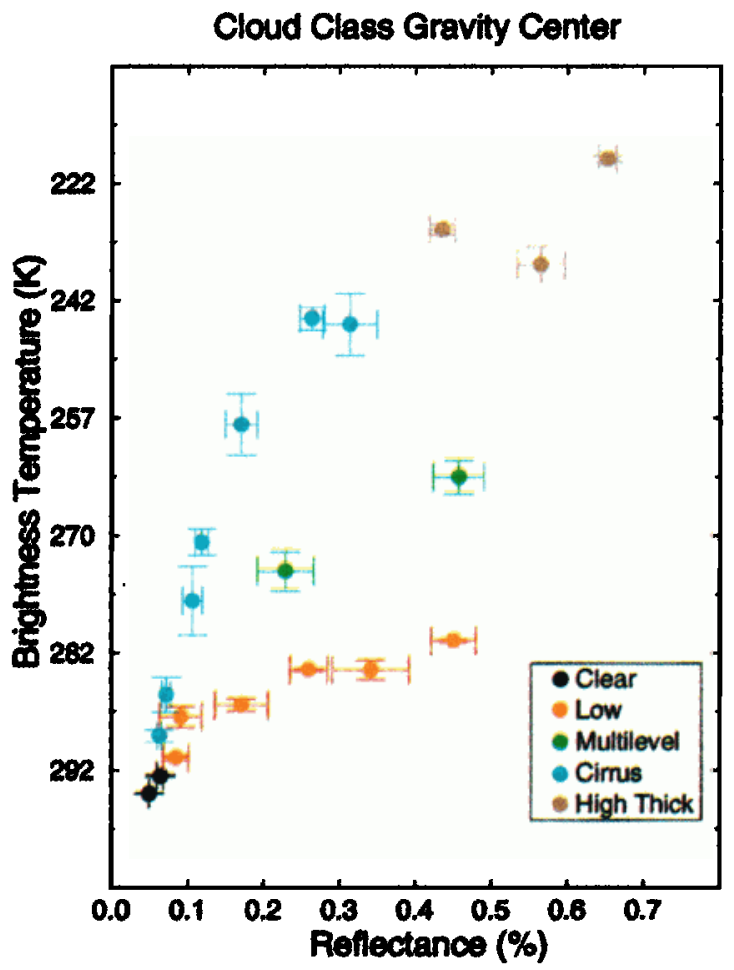

Plate 2. Positions of the cluster gravity center in the IR-VIS radiance space. The magnitude of bars centered on the gravity centers represents the value of the standard deviation parameters. occurrence frequency of clear sky, low cloud, and high-level cloud cover over the Indian Ocean are established and analyzed.

The paper is organized as follows: Section 2 introduces the data set and cloud classification method. Cloud cover analysis focusing on clear, low-, and high-level clouds is presented in section 3. In the fourth section, using the ISCCP D1 data set, the main characteristics of the clouds are given from January to March during the 1984 to 1994 period. The 1999 cloud cover

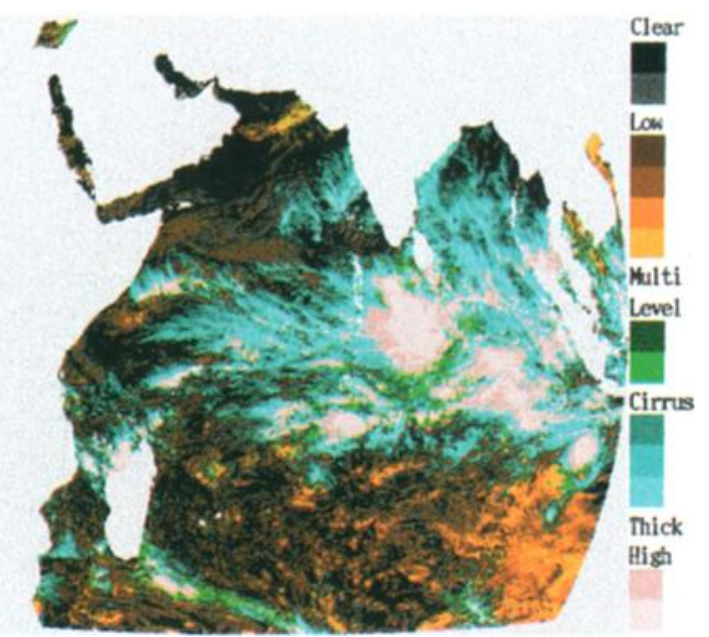

Plate 3. Cloud classification for January 23 at 0730 UTC. 
then is compared to this climatology. Conclusions and perspectives are provided in the last section.

\section{Cloud Classification Method \\ 2.1. Data and Methodology}

The present study focuses on the intensive phase period of the experiment, i.e., from January to March 1999, over the ocean region between $35^{\circ} \mathrm{N}-35^{\circ} \mathrm{S}$ and $30^{\circ} \mathrm{E}-110^{\circ} \mathrm{E}$ during the daytime. Half-hour slots have been analyzed between 0700 and 1700 local time at subsatellite point longitude $\left(63^{\circ} \mathrm{E}\right)$. Results presented here concern the slots 16 and 19 corresponding to 2400 and 1330 local time, respectively, at satellite longitude. The spatial resolution of radiance measurements is $5 \mathrm{~km}$ at the subsatellite point (the original $2.5 \mathrm{~km}$ resolution of the visible measurements is sampled to $5 \mathrm{~km}$ ). The original satellite projection is retained.

For the infrared channel the calibration coefficient provided by EUMETSAT is used to build a physically consistent data set. In the following, the computations are performed using a scale proportional to radiances, then converted to brightness temperatures. For the visible the calibration coefficient was provided by Le Borgne and Marsouin [1996] and Brisson et al. [1996], allowing to transform the counts in scaled radiances. The scaled radiances are converted to reflectances by dividing by the cosine of the solar zenith angle. As in ISCCP, restrictions have been applied to the satellite zenith angle $(\mu>0.3)$ and the solar zenith angle $\left(\mu_{0}>0.2\right)$.

To classify each pixel of this data set in a cloud type, the Dynamic Cluster Method (DCM) is used. Detailed explanation of this clustering method is given by Desbois et al. [1982] and Sèze and Desbois [1987]. As in the work of Sèze and Desbois [1987], two spectral parameters, the visible reflectance and the infrared brightness temperature, and the two associated structural parameters, namely, the local spatial dispersion (i.e., the standard deviation computed from the $3 \times 3$ values centered on the pixel), are used. Plate 1 gives an example of maps representing these parameters. For a given region (here the Indian Ocean) the Dynamic Cluster Method uses the statistical distribution of the four parameters (histogram in the fourdimensional (4-D) space of reflectances, brightness temperatures, and the two associated local spatial standard deviations) to retrieve cloud and surface types present inside the region.

The clustering step is not applied to 4-D histograms built from individual slots but to 4-D histograms cumulated over a period of 10 days for the same slot. Results at the end of this step are the center of gravity and variances of the classes. In brief, the method does not require any a priori knowledge of the classes, but a maximal number of classes (chosen as equal to 50 in the present case). To partition the histogram, the method uses an iterative process starting from randomly chosen clusters. At each iteration a new partition is built by assigning each pixel to the cluster with the nearest center gravity (according to Euclidian distance in the 4-D space of parameters). From one iteration to the next the mean distance between pixels inside classes decreases. The final partition is obtained when from one iteration to the next iteration almost the same partition is obtained and the mean distance between pixels inside classes has reached a minimum value. However, this last partition is not unique. Several initial random draws are then tried. The one which appears the best, according to the number of classes left and the value of the center of gravity, is then chosen. Then, for the chosen partition, each pixel of each individual slot is projected in the 4-D space and is assigned to the class of which it is the nearest according to its distance to the class gravity centers.

One constraint for obtaining proper results with this method is to apply it on regions where surface properties do not vary too much. In the present case, the Indian Ocean region is judged sufficiently homogeneous: surface brightness temperature remains between 299 and $302 \mathrm{~K}$ over most of the area, except the extreme southeast where it can fall to $294 \mathrm{~K}$. Outside the Sun glint area, sea surface reflectance is also relatively constant for the considered slots close to midday. It is then acceptable to apply the algorithm without segmentation in subregions. This choice simplifies the classification process.

The process has been randomly initialized for slot 16 of the period January 21-30. A solution giving 20 classes has been retained. To ensure the day-to-day diurnal stability of the classification, other slots and 10-day periods have been initialized by using the center of gravity and standard deviation of the classes obtained in the subsequent or previous slot or period as it was proposed by Raffaelli and Sèze [1995]. Although the process allows a decrease of the number of classes, this number (20) after the choice performed for the period January 21-30 stays constant over the 3 -month period. From one period to another, for the same slot, gravity center values remain relatively stable. However, large changes are observed in the gravity center values between early morning, midday, and late afternoon slots. This is due to the impact of high Sun angles on the cloud reflectance and change in the useful region of observation.

The final step is to label each class with a cloud type name. This step has been performed manually using the gravity center values of the classes (Plate 2) [Sèze and Desbois, 1987]. To confirm the class attribution performed using the gravity center values, classified images have been examined, and the compactness and space vicinity of different classes have been studied [Raffaelli and Sèze, 1995]. Finally, classes have been gathered in clear sky and four cloud types: low, multilevel and convective (convective cloud with a cloud top in the middle level), cirrus, and high thick (Plate 2). For low cloud and cirrus classes for which properties are very variable, subdivisions are used later on in the present paper (see section 2.2).

\subsection{Example of Observed Field and Classification Results}

Plate 1 gives an example of data used to construct the cloud classification. For January 23 and slot 16, Plate 1a shows a composite image of the reflectance and brightness temperature field. Different colors correspond to different brightness temperatures, and different brightnesses of colors correspond to different values of reflectance. The color legend of Plate 1 (top and right legends) shows, for two extreme levels of brightness, changes in color as a function of brightness temperature. For example, small sea surface reflectance is represented by dark color values, and very reflectant high-level clouds are represented by bright colors. Warm ocean temperatures or small cumulus clouds are shown in purple. As the cloud top brightness temperature decreases, the color turns to blue, then green, and finally yellow. Decrease of brightness temperature is due either to a decrease of the cloud top altitude, an increase of optical thickness, or an increase of cloud cover inside a pixel. The low stratiform clouds close to the Australian coast appear in blue. In the ITCZ, thin cirrus, multilayer clouds and high thick clouds associated with deep convection appear mainly in green and yellow. 
Plate $1 \mathrm{~b}$ shows for each pixel the local spatial standard deviation of the reflectance and the local spatial standard deviation of the brightness temperature computed in the $3 \times 3$ pixel square centered on the pixel. Structures that appear in this image are a useful complement of information in the cloud classification process. The principle of the color code is the same as for Plate 1a; color legends are the left and bottom legends in the color table. The dark colors indicate small values of the standard deviation of reflectance, and the bright colors indicate large values. Orange color represents a low value of the standard deviation of the brightness temperature, and green and yellow represent high values of this standard deviation. In the Arabian Sea where clear sky is prevailing, the standard deviation is low both in reflectance and brightness temperature. In the presence of small cumulus clouds the standard deviation of reflectance increases, as is the case off the Somali coast. In the south subtropical region, in particular west of Australia where large low cloud cover is present, the standard deviation of reflectance is high, and the infrared standard deviation remains relatively low. In the deep convective region the striking feature is a contrast between the high standard deviation of dark high-level clouds and the low standard deviation of the bright high-level clouds in infrared.

Projection of the cluster gravity center for slot 16 (2400 LT at satellite longitude) and over a 3-month period is shown in Plate 2. The $y$ axis represents brightness temperature, and the $x$ axis represents the reflectance. The well-known shapes in the reflectance/brightness temperature space corresponding to cirrus with varying emissivity and low cloud cover with varying coverage inside the pixel [Platt, 1983; Sèze and Rossow, 1991; Minnis et al., 1993] are observed in this plate. Starting from the darkest and warmest clusters, the orange branch shows the progression from clear sky to small cumulus and thick low cloud deck, and the turquoise and pale brown branch follows the progression from thin cirrus to cirrus and high thick clouds. The magnitude of the two bars centered on each gravity center gives the visible and infrared standard deviation parameter values. We remark that the ratio between these parameter values changes between the class labeled cirrus and the classes labeled low clouds. This is important in the labeling process in particular to help in the separation of very thin cirrus from very small broken low cloud [Stubenrauch et al., 1999]. The two classes labeled multilevel clouds from their position in the brightness temperature/reflectance diagram could be interpreted as well as medium-level clouds or multilayer cloud. However, the relatively high standard deviation in brightness temperature of the brightest of these two classes and its spatial organization in the classified images are indicative of its multilayer structure.

In the present study, a further step has been added to the classification process to improve the separation of pixels covered by very thin cirrus from those covered by small broken low cloud. For pixels belonging to the two cirrus classes the closest to clear-sky class, a neighboring test has been added. If in the eight pixels next to the considered pixel there is a pixel belonging to the other cirrus class or high thick cloud class (one of the eight remaining classes, Plate 2), the pixel is attached to the cirrus class; otherwise this pixel is attached to the low cloud classes. With this test, $2 \%$ of the cirrus pixels are reclassified into the broken low cloud type classes. These pixels are boundary pixels between fully clear-sky pixels and broken low cloud pixels.

Two clusters have been labeled as clear sky in Plate 2. However, if we compare the gravity center of these two classes, one of them is $1 \mathrm{~K}$ colder, the reflectance is slightly larger, and the two associated local standard deviations are also larger. Visual inspection of the composite images such as Plate $1 \mathrm{a}$ and analysis of the space vicinity of that class in the classification images indicate that often small cumulus must be present in the pixel. The cloud cover inside the pixel will be almost $0 \%$, but cannot be labeled fully clear. Therefore this class will be called clear to broken low class (CL_BL). The decrease of $1 \mathrm{~K}$ between the fully clear pixel class (CLEAR class) and the CL_BL class (large if it is due to the impact of very small cumulus) is due to the fact that the CL_BL class is more frequent in the south of the Indian Ocean than in the northern part. It is the opposite case for the CLEAR class. The radiative impact of cloud inside the CL_BL class must be negligeable. However, for study on cloud life cycle and on the impact of aerosols on the formation of low clouds, the CL_BL class has to be separated from the CLEAR class.

In the following, the so-called low clouds are considered to have no higher overlapping cloud layer, the exception being very thin cirrus above low cloud. The multilayer cloud must be a mixing of low-level clouds covered by cirrus, middle-level cloud topped with convective cloud, or middle-level cloud covered by thin cirrus. Addition of the water vapor channel [Roca et al., this issue] or of a parameter taking into account the simultaneous variations of visible and infrared radiances on a local spatial domain [Raffaelli and Sèze, 1995] could help in the differentiation of these cases.

Plate 3 shows the classification obtained for January 23 at 2400 local time at the satellite longitude (slot 16). Clear sky is in dark, low clouds are in brown and orange, cirrus are in turquoise, and high thick clouds are in pink and white. The color code used in this image is based on the color code used in Plate 2. However, the color level number is 13 instead of 5 . This is to allow discrimination between classes of a same cloud type in function of their reflectance (and brightness temperature). The darkest is the tone in a range of color, and the smallest is the reflectance of the gravity center of the class (Plate 2). To obtain 13 classes from 20 classes, some of them have been grouped together. For low clouds the dark brown represents the darkest class, and the lighter brown shows the next two classes in the increasing reflectance scale. The light orange represents the two brightest low cloud classes. For cirrus, dark turquoise represents the cirrus class with the smallest reflectance, and the intermediate turquoise represents the next three cirrus classes in the reflectance scale. Light turquoise represents the three classes with the largest reflectance. For thick high-level clouds, dark pink represents the two classes with the smallest reflectances.

To clarify the statistics presented in section 3 , an additional grouping of classes is performed. The three low cloud classes with the smallest reflectances are labeled together as broken low cloud. Thick low clouds correspond to the remaining three classes. The four cirrus classes with the smallest reflectance are labeled thin cirrus. Thick cirrus corresponds to the remaining three classes. In spite of the specific characteristics of each class, each pixel is considered as fully covered or fully clear in the statistics below.

\section{Climatology Over the 3-Month Period}

\subsection{Mean Frequency of Clear Sky and Clouds}

Over the 3-month period and the $35^{\circ} \mathrm{N}-35^{\circ} \mathrm{S}$ and $30^{\circ} \mathrm{E}-$ $110^{\circ} \mathrm{E}$ region, the occurrence frequency $(\mathrm{OF})$ of clear sky $(\mathrm{COF})$, low cloud classes (LOF), multilayer cloud classes (MOF), and cirrus plus high thick cloud classes (HOF) are estimated using our 


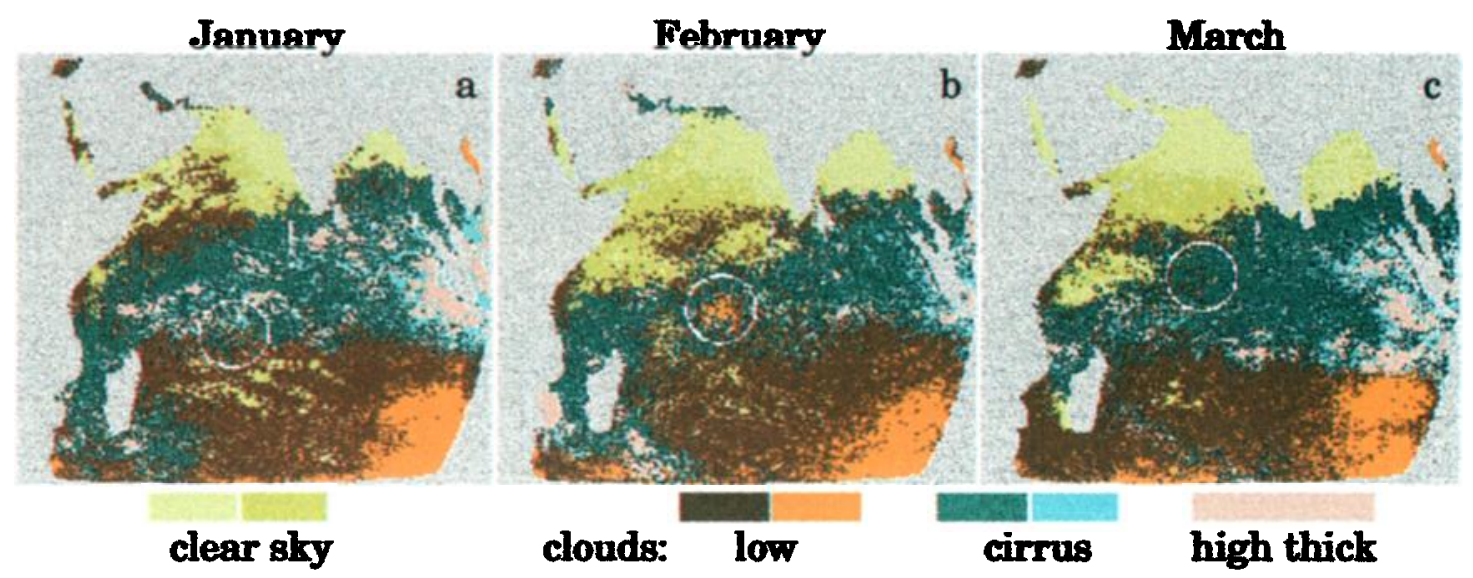

Plate 4. The most frequent class occurrence (clear sky and low, cirrus, and high thick clouds) averaged over 1-month periods. (a) January, (b) February, and (c) March 1999.

classification. The OF are also estimated for broken low cloud, low cloud, thin cirrus and thick cirrus, and high thick cloud subclasses. The occurrence frequency (OF) is defined as the ratio of the number of pixels belonging to certain classes over the total number of pixels. In other words, OF in the following do not represent cloud cover estimate per se, but rather amount of given classes over a given region during a given period. The slot used is slot 16 (2400 LT at satellite longitude).

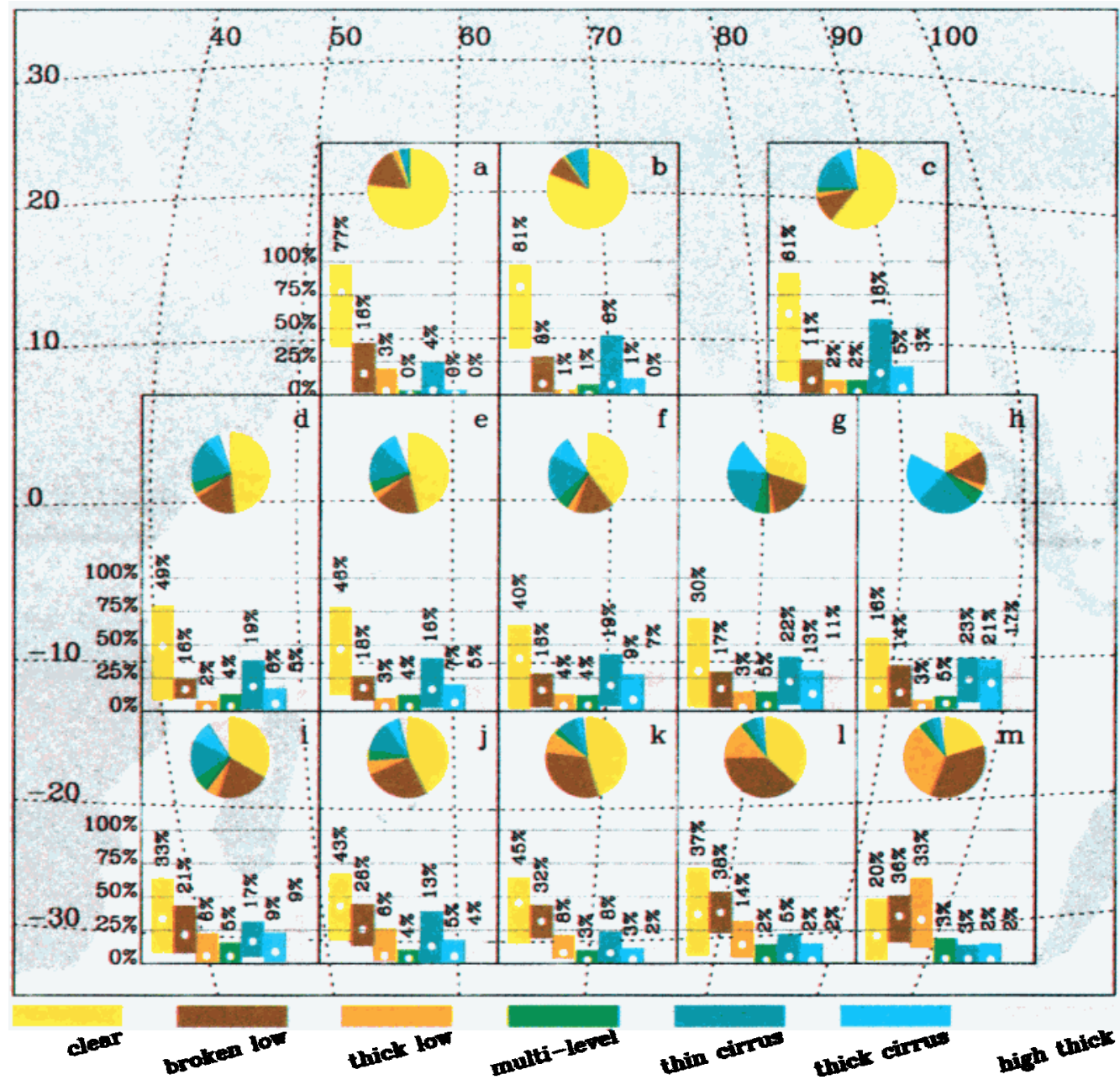

Plate 5. Occurrence frequency of cloud type class for the 3-month period and 13 subregions over the Indian Ocean (continental parts are not taken into account). The color bars span between the lowest and the highest values of the occurrence frequency of each cloud type class. Mean values of the occurrence frequency are reported and presented by the white circles in the color bars. The pie charts show the mean values of the frequency occurrence. 
Table 1. Frequency of Occurrence of the Different Cloud Types for January, February, and March

\begin{tabular}{lcccccccc}
\hline Month & $\begin{array}{c}\text { Clear, } \\
\%\end{array}$ & $\begin{array}{c}\text { Clear to } \\
\text { Broken, } \%\end{array}$ & $\begin{array}{c}\text { Broken } \\
\text { Low, \% }\end{array}$ & Low, \% & $\begin{array}{c}\text { Multilevel, } \\
\%\end{array}$ & $\begin{array}{c}\text { Thin Cirrus, } \\
\%\end{array}$ & $\begin{array}{c}\text { Cirrus, } \\
\%\end{array}$ & $\begin{array}{c}\text { High Thick, } \\
\%\end{array}$ \\
\hline January & 27 & 12 & 21 & 8 & 4 & 14 & 8 & 6 \\
February & 32 & 14 & 21 & 7 & 3 & 12 & 6 & 4 \\
March & 27 & 13 & 20 & 7 & 4 & 15 & 8 & 7 \\
\hline
\end{tabular}

Table 1 gives these statistics for each month from January to March for the entire region. Statistics for January and March are very close. In February there is a decrease in cirrus and high thick cloud cover and an increase of clear sky. The OF of fully clear pixels and low cloud pixels are almost the same (28\%) for the whole period. Compared to this, the CL_BL OF is large (13\%). Over the 3-month period the OF of high-level cloud (cirrus plus high thick) is $27 \%$, and what we called multilevel cloud is only $4 \%$.

\subsection{Most Frequent Cloud Cover Type}

At each pixel level a hierarchical classification has been applied (Plate 4). This three-step process determines the following for each pixel: (1) if the OF of clear sky is greater than the OF of cloudy sky or not, (2) for the cloudy cases if LOF is greater than HOF or not, and (3) for low cloud case if the OF of broken cloud is greater than the OF of low cloud deck and for high level cloud case what is the maximum OF between thin cirrus, cirrus, and high thick clouds.

The CL_BL class is not taken into account in the first step of this process which determines if the pixel is more frequently clear or more frequently cloudy. The effect of that is to assume that the pixels of the CL_BL class are equally distributed between the clear class and the cloud class. Then, if the result of the first step is clear, this class is attached to clear allowing a subdivision of this class (see next paragraph), and if the result of the first step is cloud, this class is attached to broken low cloud increasing the weight of the low cloud in step 2 and the weight of broken low cloud in step 3 .

Plate 4 presents results of this hierarchical classification for each of the 3 months. Clear sky is in yellow, broken low clouds are in brown, low clouds are in orange, thin cirrus and cirrus are in dark turquoise and turquoise, and high thick clouds are in pink. The white circle points out the Sun glint region. In this last region, results must be interpreted with caution (clear sky and some thin cirrus can be underestimated, and low clouds can be overestimated by our classification). For clear sky, if the occurrence frequency of CL_BL is less than $30 \%$ of the CLEAR class, the color is light yellow, and if the occurrence frequency of CL_BL is greater than $30 \%$ of the CLEAR class, the color is bright yellow.

Structures are zonal in Plate 4. Clear sky occurs frequently in the northern part of the Indian Ocean, over the Bay of Bengal, and over the Arabian Sea. Low clouds are prevalent in the southern part. High-level clouds, related to the Intertropical Convergence Zone, spread from Indonesia to the north of Madagascar. High thick clouds related to deep convection occur frequently near Indonesia. There is another region of frequent high-level cloud occurrence, starting from the African coast at the Madagascar latitude and oriented northwest to southeast (as can be verified by looking at METEOSAT image animation). This feature appears similar, although much weaker, to the South Pacific Convergence Zone (SPCZ) and may be named South Indian Convergence Zone (SICZ).
We note a decrease in high-level cloud occurrence in February in the ITCZ, when the frequency of high-level cloud systems escaping from Mozambique toward the southeast increases. In March, near Indonesia, the region of frequent highlevel cloud occurrence related to deep convection region spreads south. From January to March the clear sky becomes more and more prevalent over the Arabian Sea as already observed by Bony et al. [2000].

\subsection{Regional Distribution}

The entire Indian Ocean region is currently divided into subregions. For these subregions, Plate 5 shows the occurrence frequency of the cloud type class for the 3-month period. The color bars in each region show the extent of the January to March distribution of the daily occurrence frequency of each class in the region. In this plate, clear sky represents CLEAR plus CL_BL class occurrence frequency. In the north, clear-sky occurrence frequency is at least $60 \%$ in the Bay of Bengal (region c) and $77 \%$ in the Arabian Sea (regions a and b). Near the coast of India in region $b$ this percentage can reach $100 \%$ (results not shown). Off the coast of Indonesia (region h), cirrus clouds and high thick clouds occur 61 and $17 \%$ of the time, respectively. On certain days the high thick cloud cover percentage can reach $45 \%$. In the ITCZ and the southern part of the Indian Ocean, clear sky is not the most frequent situation, and on certain days this percentage can fall to almost $0 \%$ for regions $f, g, h$, and $m$. However, this percentage on a certain day can also be as high as $\mathbf{5 0 \%}$. In the southeast subtropics, low cloud occurrence frequency reaches $69 \%$ (region $\mathrm{m}$ ).

\subsection{Frequency of Clear Sky, Low- and High-Level Cloud}

Plate 6 shows the OF of the CLEAR class (Plates 6a, 6b, and 6c), the LOF where the CL_BL class has been taken into account (Plates 6d, 6e, and 6f), the LOF without the CL_BL class (Plates 6g, 6h, and 6i), and the HOF (Plates 6j, 6k, and 6l) for each month. As in Plate 4, the white circles are centered on the Sun glint region. In Plate 6, clear-sky OF represents the occurrence frequency of the CLEAR class only, and the CL_BL class has not been taken into account. This explains the differences which can be observed between Plates 5 and 6 for clear-sky occurrence frequency.

We again note the increases of clear-sky OF from January to March in the Arabian Sea and the decrease of LOF. What was not observable in the previous plates is the lack of clear sky in the eastern and southern parts of the Indian Ocean in March. The strong increase of OF values when the CL_BL class is added to the LOF is obvious in Plates 6d-6i, in particular near Arabia and Somali. In March the decrease in HOF south to Madagascar yields an increase in LOF. This increase in LOF can also be due to a real increase in the low cloud cover or to the absence of high thick cloud cover allowing the observation of the low cloud deck. In the ITCZ we observe again the large variation of HOF from one month to another, which may be related to the Madden-Julian oscillation. 

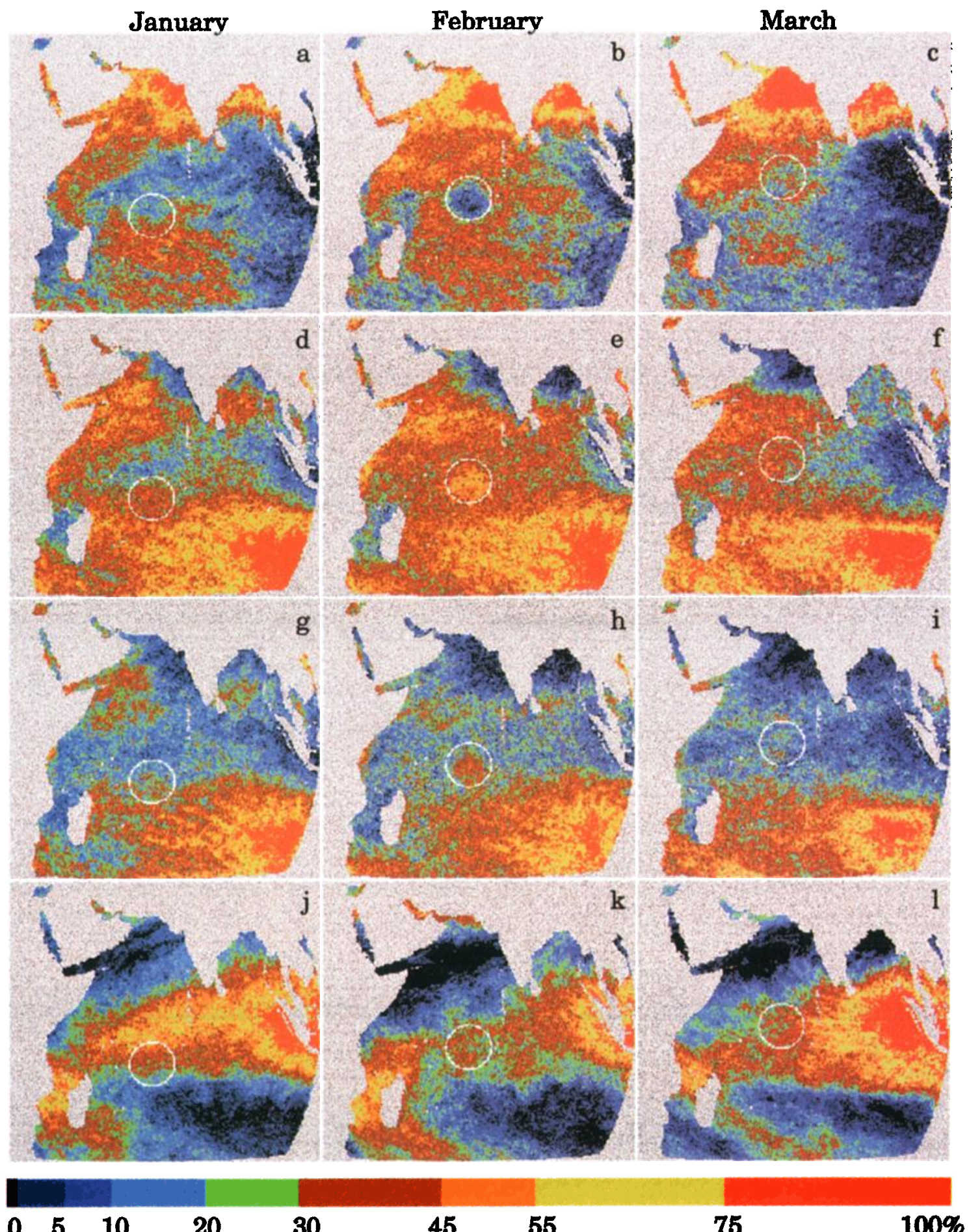

45

55

75

$100 \%$

Plate 6. ( $a, b, c)$ Frequency occurrence of the clear class, (d, e, f) low clouds where the CL_BL class has been taken into account, (g, h, i) low clouds without the CL_BL class, and (j, k, l) high-level clouds.

\section{Comparison With ISCCP D1 Data}

\section{ISCCP D1 Data}

The International Satellite Cloud Climatology Program (ISCCP) data set provides a global coverage of cloud cover properties (coverage, cloud top pressure, and optical thickness) [Rossow and Schiffer, 1999] using infrared and visible imagery observed by operational meteorological satellites
[Rossow et al., 1996]. The original data, radiance measurements over fields of view ranging from 4 to $10 \mathrm{~km}$ in size in IR, are sampled to a resolution of about $25-30 \mathrm{~km}$. The visible radiances are first averaged to the IR radiance resolution. The algorithm applied at the pixel level is a two-stage process: (1) cloud identification and (2) cloud properties retrieval. Cloudy pixels are classified in cloud types according to cloud top pressure and cloud optical depth. Forty-two cloud types are defined 


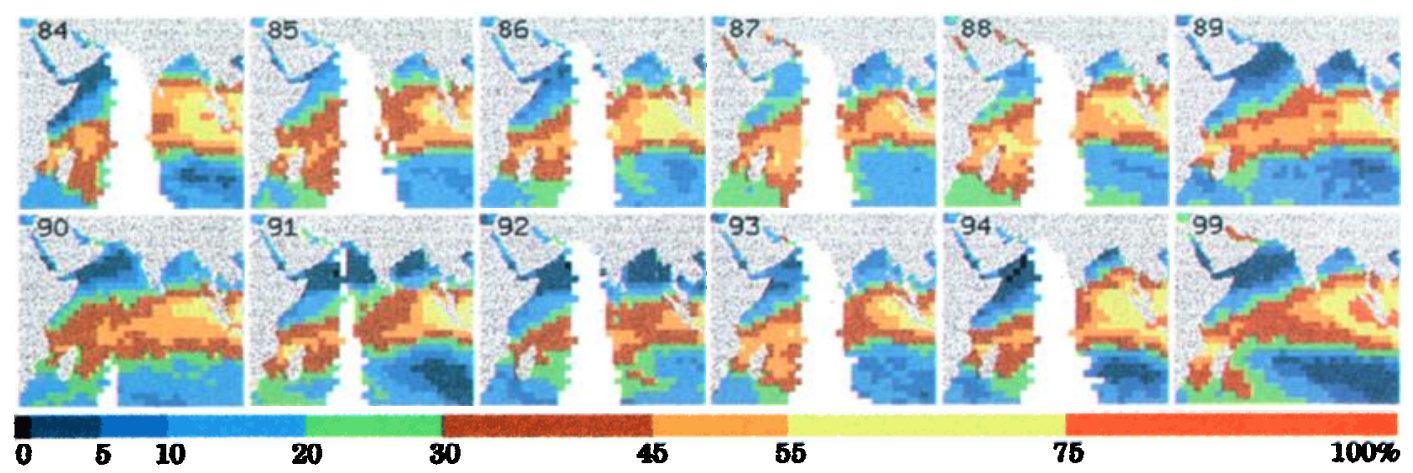

Plate 7. High-level clouds occurrence frequency averaged over January-March for ISCCP (1984-1994) and METEOSAT (1999).

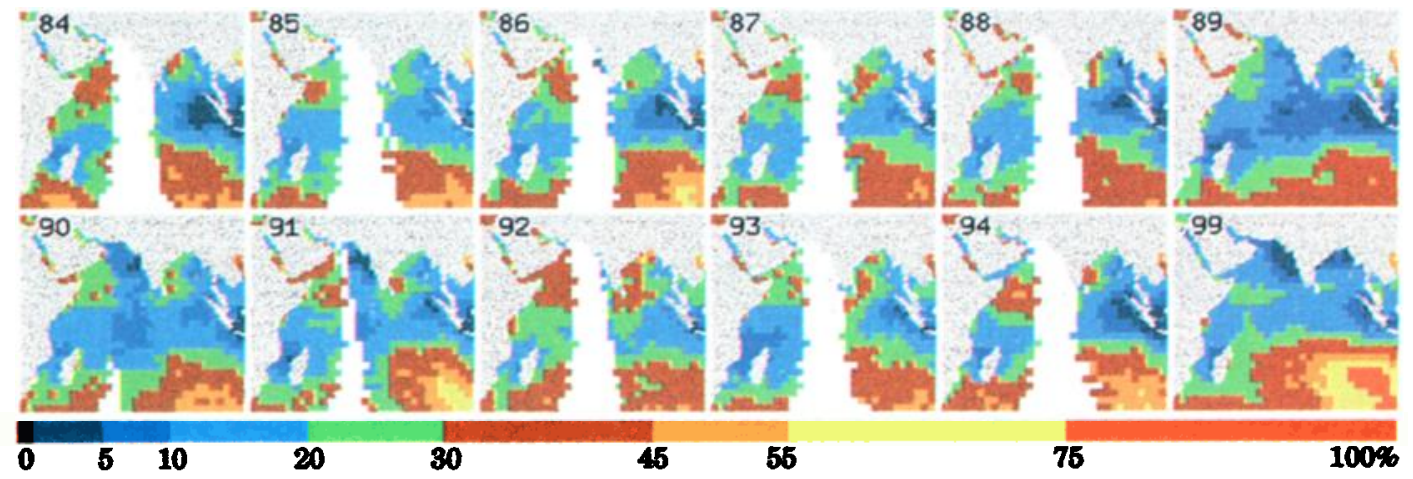

Plate 8. The same as in Plate 7, but for low clouds.

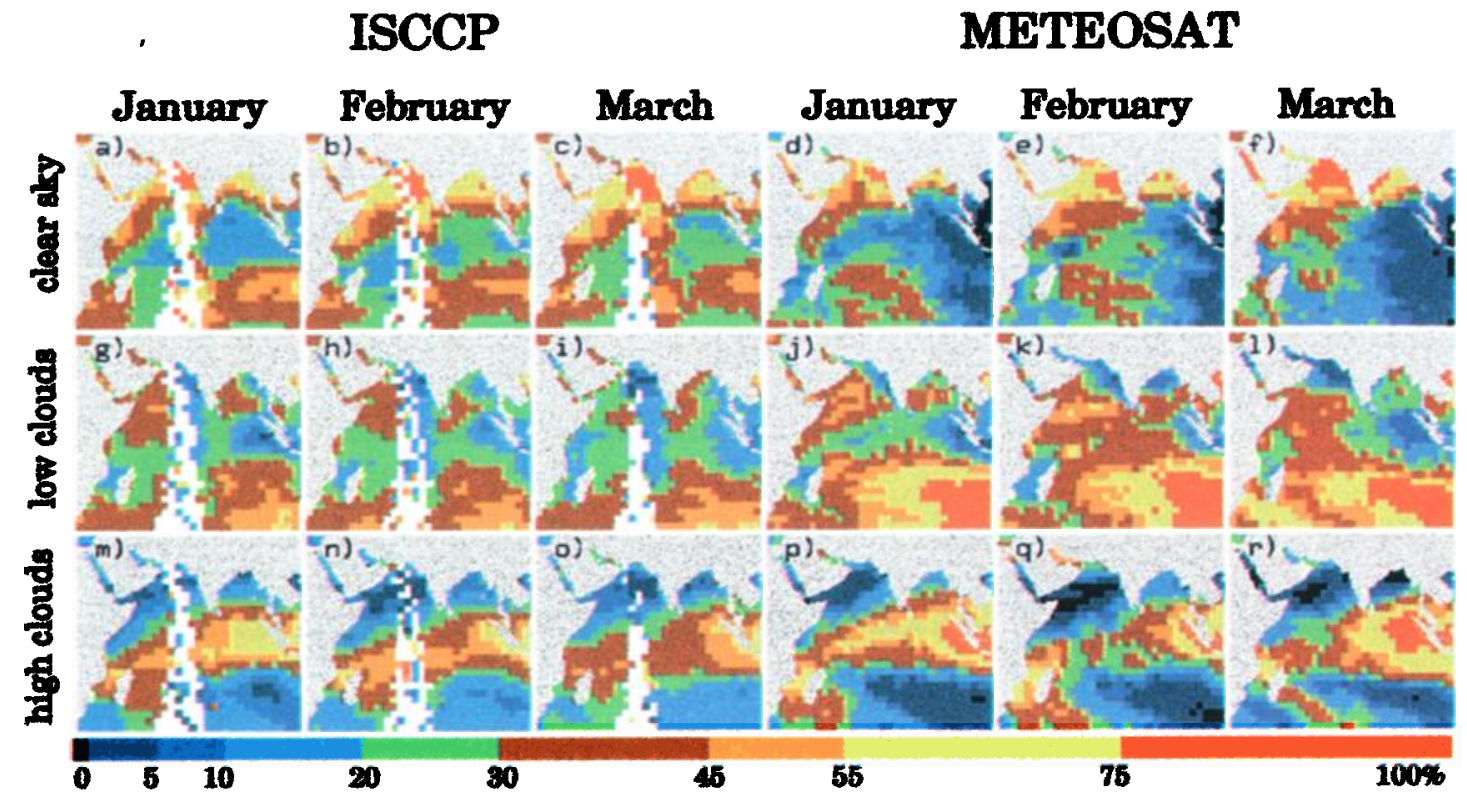

Plate 9. Clear-sky, low- and high-level clouds occurrence frequency for January, February, and March for ISCCP (average over 1984-1994) and METEOSAT (1999). 
using seven ranges in pressure and six ranges in optical depth. The results in the D1 data set are assembled in the form of cloud type distributions and mean properties at the $280 \mathrm{~km}$ by $280 \mathrm{~km}$ scale. The use of a geostationary satellite allows a time resolution of 3 hours in a large part of the globe, except in the central part of the Indian Ocean between $60^{\circ}$ and $80^{\circ} \mathrm{E}$. Throughout this region, the INSAT geostationary satellite has been available in the ISCCP project only between April 1988 and March 1989. The rest of the time period has been filled in with data from the advanced very high resolution radiometer (AVHRR) on board the NOAA polar orbiters. The AVHRR data are available twice or four times a day depending on the time period.

For each of the 3 months, January, February, and March, and each year in which ISCCP D1 data were available (1984 to 1994), total cloud cover and cloud type cover were extracted for the $35^{\circ} \mathrm{N}-35^{\circ} \mathrm{S}, 30^{\circ} \mathrm{E}-110^{\circ} \mathrm{E}$ region at $0900 \mathrm{UTC}(1330 \mathrm{LT}$ at METEOSAT-5 satellite longitude). To avoid erroneous information owing to the lack of data in the $60^{\circ} \mathrm{E}-80^{\circ} \mathrm{E}$ band, statistics are retained only for grid boxes with 25 days of data over the 28 to 31 days of the month. The other grid boxes are marked as missing. The original grid is projected on a $2.5^{\circ} \times$ $2.5^{\circ}$ latitude-longitude grid. Rather than referring to cloud cover percentage, it is preferable, as in our study with DCM applied to METEOSAT 1999 data, to refer to the occurrence frequency of cloudy or clear pixel over a given period and a given spatial scale. In the present case, the scale is $280 \mathrm{~km}$ by $280 \mathrm{~km}$, and the time period is 1 or 3 months. As in our classification, this classification represents a classification of cloud top estimated from VIS and IR radiances measured from a space platform. At pixel scale, only one cloud type is retrieved even if several cloud layers are present.

\subsection{Comparison}

4.2.1. Main spatial features. To compare DCM 1999 cloud field with the ISCCP 1984 to 1994 cloud field, OF maps obtained with the classification performed with slot $19(0900$ UTC) are averaged to the $2.5^{\circ} \times 2.5^{\circ}$ latitude-longitude grid. Plate 7 presents an average of the ISCCP HOF maps for the January to March period of each year between 1984 and 1994, and of the DCM HOF maps for the January to March 1999 period.

As it is defined in ISCCP, high-level cloud OF are obtained from the D1 data by summing the OF of cloud types with cloud top pressure less than $440 \mathrm{hPa}$. The year 1989 is the only year in which INSAT data are used in ISCCP. For the other years the area in white indicates regions in which data are missing, meaning less than 75 days of data are available over the 3-month period. There are more data for this region in 1990 and 1991 than for the other years. For these years, structures appearing in this region must be interpreted with caution. In Plate 7, in the DCM map from 1999 as well as in the ISCCP maps from 1984 to 1994 , the ITCZ is well marked by an OF of high-level cloud greater than $30 \%$. In the region of deep convection off the coast of Indonesia (region $h$ ), HOF are often larger than $55 \%$. Latitudinal extension of this HOF peak is variable from one year to the next. The variations between January, February, and March HOF maps of the same year (not shown) are of the same order as those observed from one year to the next in Plate 7. However, the January, February, and March HOF maps averaged over the 1984-1994 period (Plates 9m, 9n, and 9o) show that near Indonesia during this period the OF of high thick cloud related to deep convection is larger in January than in February or March. In Plate 7 the structure that we called SICZ is a relatively stable structure throughout the year. However, its spreading south to Madagascar is year-dependent.

Plate 8 is similar to Plate 7, but for the LOF. As it is defined in ISCCP, low cloud OF are obtained from the D1 data by summing the $\mathrm{OF}$ of cloud types with cloud top pressure greater than $680 \mathrm{hPa}$. For 1999 the DCM LOF maps do not include the OF of the CL_BL class. In the Arabian Sea and the Bay of Bengal (regions a, b, and c), DCM LOF is smaller than ISCCP LOF. On the contrary, in the southeastern subtropics (regions 1 and $\mathrm{m}$ ), the LOF is greater in the DCM 1999 map than in the 1984-1994 ISCCP maps. If the OF of CL_BL class is added to the LOF, LOF becomes greater over the northern regions in the DCM maps compared to the ISCCP maps (Plates $9 \mathrm{~g}, 9 \mathrm{~h}$, $9 \mathrm{i}, 9 \mathrm{j}, 9 \mathrm{k}$, and $9 \mathrm{l}$ ). In the southeastern subtropics the gap between the DCM and ISCCP LOF increases.

Plate 9 shows that the less low cloudiness observed in ISCCP data than in METEOSAT 1999 data is compensated for by a greater COF. In Plate 9, COF for METEOSAT corresponds to the occurrence frequency of the CLEAR class without the CL_BL class as in Plates 6a-6c. The LOF corresponds to the occurrence frequency of low cloud class plus the CL_BL class as in Plates 6g-6j.

4.2.2. Regional analysis. For the 13 regions defined in Plate 5, average values over the regions of HOF, LOF, and COF for the January to March period have been computed for ISCCP and DCM (Figures 1a, 1b, and 1c). For ISCCP, average values have been computed for each year separately, and then statistics have been extracted from the 11-year distribution of average values. These statistics are the maximum and minimum value, the mean value, the standard deviation, and the median value. In Figure 1 the vertical line for each region delineates the range of value of the 11-year distribution, the left horizontal line indicates the mean value, and the right horizontal line indicates the mediane value. The standard deviation is represented with a triangle. In clear-sky and low cloud graphics (Figures 1b and 1c) for DCM the circle represents the average values obtained without taking into account the CL_BL class, and the star represents the average values obtained taking into account the CL_BL class. For ISCCP a test requires at least 25 days of data over the month for a grid box. Another test has been added here, requiring that these 25 days of data be obtained from a geostationary satellite (METEOSAT for the western part of the region and GMS for the eastern part of the region). A last test is performed to take into account only grid boxes which have information for the 11 years. The 1999 DCM average values are also computed using only the grid box obtained after this last test. Because of these last tests, no statistics are reported for regions $b, f$, and $k$ in Figure 1.

In Figure 1a the regional tendencies of DCM HOF and ISCCP HOF are in agreement. The DCM OF are generally in the range of scale of ISCCP HOF during the 1984-1994 period. The maximal value of HOF for ISCCP and DCM and the range of variability the most extended (35\%) for ISCCP are reached for the region near Indonesia (region h). DCM 1999 HOF reaches the largest values of ISCCP HOF for the 1984 to 1994 period. The relatively frequent presence of high-level cloud between Africa and Madagascar is not well represented in Figure 1a. This is due to the fact that the structure that we called SICZ falls between regions $d$ and $i$.

In the southeastern subtropics the gap between the DCM 
a) High Clouds

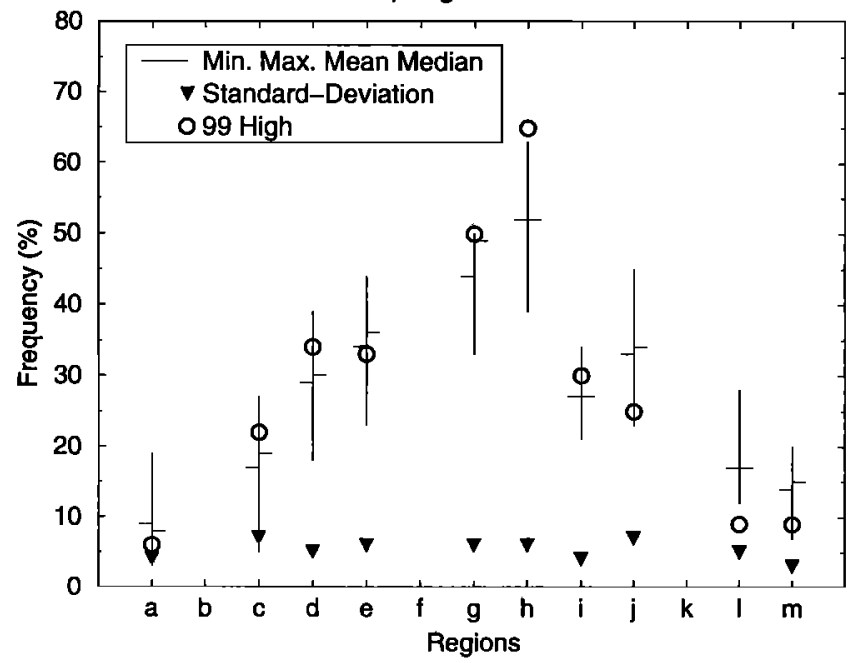

c) Clear Sky

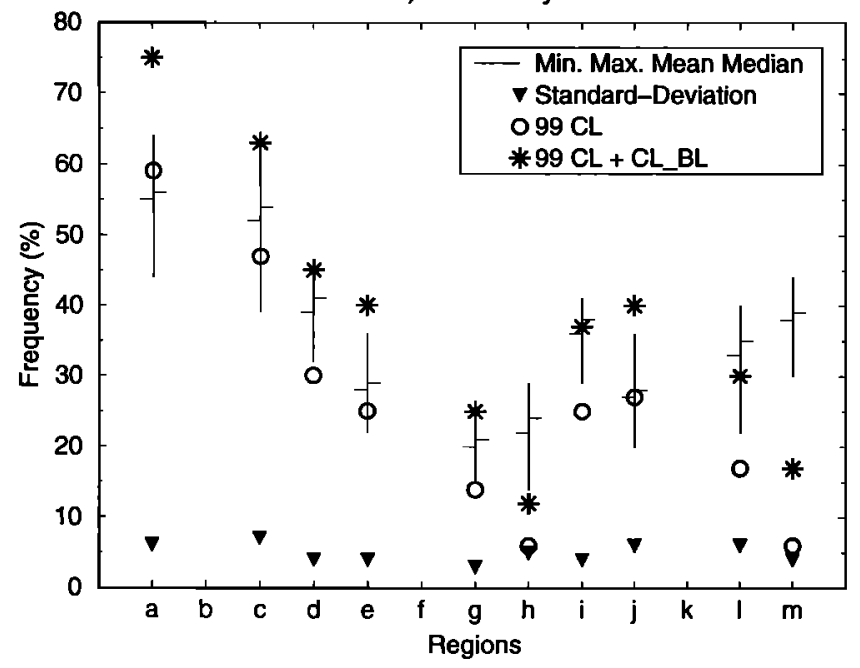

b) Low Clouds

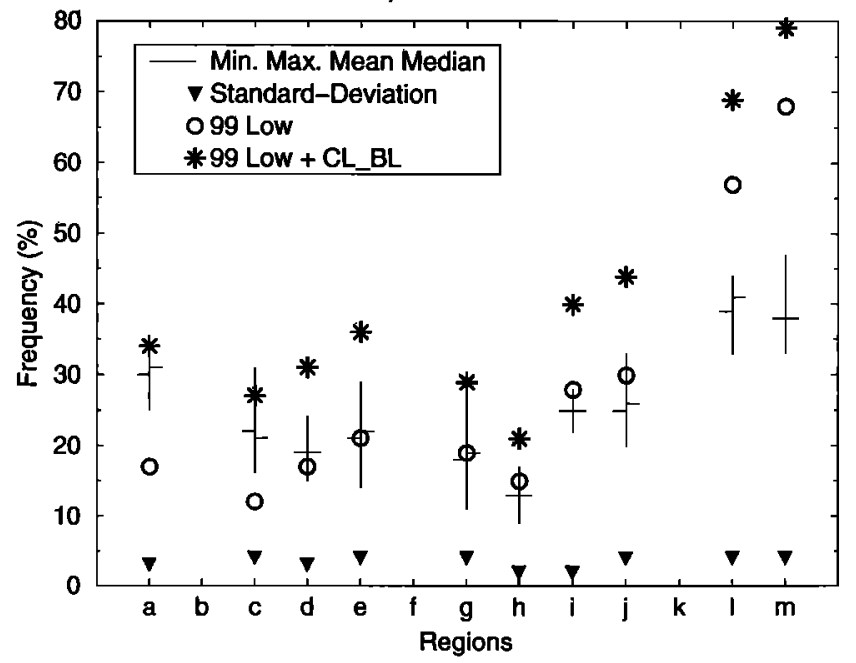

d) Low + Middle or Multilayer Clouds

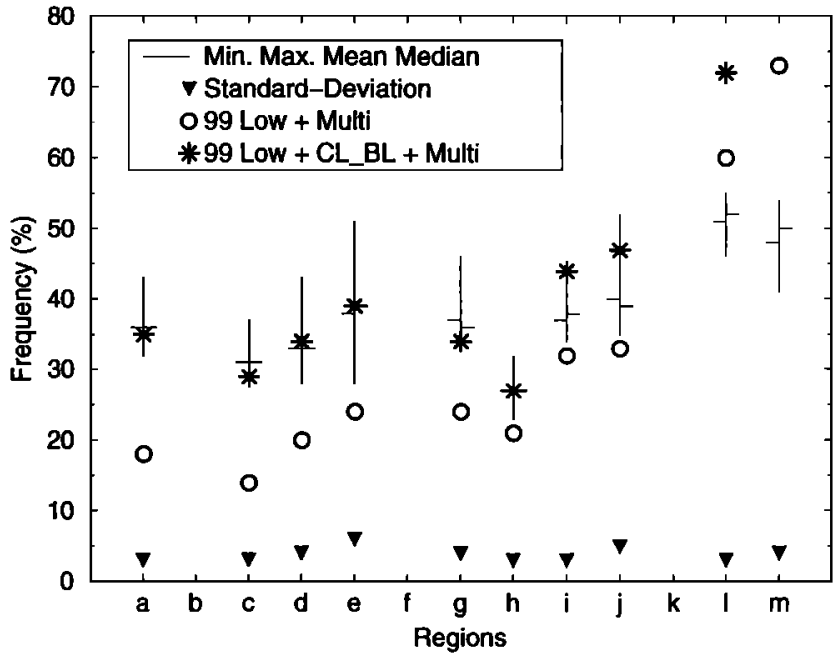

Figure 1. Average values of frequency occurrence of (a) high-level clouds, (b) low-level clouds, (c) clear sky, and (d) low plus midlevel clouds for the January to March period of 1984-1994 for ISCCP analysis (the continuous-vertical line delineates the range of values of the 11-year distribution, the left horizontal line indicates the mean value, and the right horizontal line indicates the median value), and DCM analysis for 1999 (circles and stars; see text for more details). Triangles show standard deviation for the ISCCP analysis.

and ISCCP LOF increases. Figure $1 b$ confirms these observations. In Figure $1 \mathrm{~b}$ the two DCM values (LOF with and without the CL_BL class) flank the range of ISCCP LOF values or at least a part of it, as expected for the two more southeastern regions (regions 1 and $\mathrm{m}$ ). For the COF statistics the same behavior is observed in Figure 1c. A previous comparison of clear-sky pixel percentage retrieved by the DCM and the ISCCP algorithm was performed during the EUCREX experiment over the North Atlantic [Raffaelli and Sèze, 1995]. It was also found that the clear-sky pixel percentage given by ISCCP is larger than the fully clear-sky pixel percentage given by DCM, and the difference found between the two percentages is lower than the CL_BL class percentage. In this study, the two algorithms were applied on the same data set. Thus it can be thought that CLEAR OF is a low bound and CLEAR plus CL_BL OF is a high bound for what could be found with ISCCP for 1999. However, in Figure 1a for regions a and m, DCM 1999 COF appears to be larger and lower than could be expected from the ISCCP COF 1984 to 1994 values, respectively. In these two regions a and $\mathrm{m}$, sea surface temperatures compared to the average value of sea surface temperature over the Indian Ocean are larger and smaller, respectively.

In the ISCCP cloud detection algorithm, brightness temperature threshold used is pixel-dependent. The DCM algorithm (see section 2) has been applied to the pixel parameter 4-D distribution (brightness temperature, reflectance, and local spatial standard deviation of these two parameters) cumulated over the whole region to define cloud class gravity center, this allowing after that to classify each individual pixel. To answer the question, Does the change in surface brightness temperature between the north and southeast regions influence results of DCM and explain the bias found for regions 1 and $\mathrm{m}$ ?, DCM has been applied separately to several of the regions, and COF have been computed for these regions using these new regional classifications. The COF obtained with the regional classifications are equal to the previous COF plus or minus $4 \%$, except 
for region $\mathrm{m}$. For this region, $\mathrm{COF}$ increases by $6 \%$, but the DCM COF still remains lower that could be expected from the 1984-1994 ISCCP COF statistics. For region a, COF decreases by $4 \%$.

For the LOF another source of incertitude to interpret the differences observed between the ISCCP OF and the DCM OF is the separation between low cloud and upper-level cloud, especially the middle-level cloud for ISCCP and that we have called multilayer cloud for DCM. In the DCM classification the cloud type label is given by not only using the brightness temperature but also by using the reflectance and the two associated structural parameters. In ISCCP the separation is related to a strict threshold in estimated cloud top pressure. Moreover, for persistent marine stratus, due to systematic errors in the vertical temperature profiles used to estimate cloud top pressure from the cloud top brightness temperature, ISCCP cloud top pressures [Rossow and Schiffer, 1999; Stubenrauch et al., 1999] are too low by 50-80 mbar. We have tested the effect of adding occurrence frequency of clouds belonging to the 680 and $560 \mathrm{hPa}$ rank in the computation of ISCCP LOF. To add this pressure level increases the regional ISCCP LOF by $8 \%$ at the most (regions $\mathrm{d}, \mathrm{e}, \mathrm{g}$, and $\mathrm{j}$ ). In the southeastern subtropics, ISCCP LOF are still smaller than DCM 1999 LOF.

Figure $1 \mathrm{~d}$ gives the OF of low cloud plus midlevel clouds for ISCCP and low cloud plus the multilayered cloud for DCM. In ISCCP, cloud top temperature is determined using the IR brightness temperature and the reflectance making the assumption of a single cloud layer covering the whole pixel. In DCM, certain classes, due in particular to the center gravity value of the local spatial standard deviation parameters, are labeled multilayer cloud cover. However, the gravity center of these classes by their properties in the brightness temperaturereflectance space would be classified as middle-level clouds in the ISCCP algorithm. The OF of low plus middle-level cloud for ISCCP and low cloud plus multilayered cloud will be both called LMOF. The DCM LMOF when the CL_BL OF is included fall inside the range of ISCCP LMOF. Except for regions 1 and $\mathrm{m}, \mathrm{DCM}$ LMOF are never larger than ISCCP LMOF.

In Figure 1 the maximal variations of HOF and COF between 2 different years represented by the extent of the vertical line are in average over the 13 regions of $16 \%$. The maximal variations of LOF and MOF are in average of $12 \%$. The OF of the CL_BL class is in average over the 13 regions of $12 \%$. In ISCCP, ocean cloud amounts are expected to be accurate to within $\pm 9 \%$ [Rossow and Garder, 1993].

In summary, the DCM COF and DCM COF plus the CL_BLOF give a low and high bound for the COF which could be estimated by ISCCP for 1999. Compared to the 1984 to 1994 period, the DCM 1999 COF is high for region a in the Arabian Sea. It can be a real change in cloud cover. However, in this region of very frequent occurrence of clear sky and aerosols [Leon et al., this issue], it can be due also to the detection of aerosols as a cloud in ISCCP [Rossow and Shiffer, 1999]. The LOF is abnormally high in 1999 in the southeastern Indian Ocean (region $\mathrm{m}$ ) compared to the LOF during the 1984 to 1994 period. Near Indonesia (region h) the HOF is large compared to the mean value obtained with the ISCCP 11 years. In the ITCZ the DCM MOF is relatively low compared to the ISCCP MOF. Some of the DCM high-level cloud pixels could have been labeled middle-level cloud by ISCCP.

\section{Summary and Conclusions}

Cloud cover observed with METEOSAT-5 during the INDOEX experiment is analyzed using the classification technique developed by Sèze and Desbois [1987] and Raffaelli and Sèze [1995]. For January to March 1999, maps of cloud cover type are created for each day at 0730 and 0900 UTC. For the 3-month period and for each individual month the distribution of cloud cover type is analyzed. For the same months the climatology of clear-sky, low cloud, and high cloud occurrence frequency from ISCCP between 1984 and 1994 is presented. This climatology and our results for 1999 are compared.

The main cloud types observed with DCM and applied to METEOSAT data during the period from January to March 1999 are globally in agreement with previous observations of the climate regimes present in the region: north and south oceanic subtropical subsidence zones and Intertropical Convergence Zone. In the north subtropics the frequency of clear sky and very small cumulus is prevalent, in the tropics highlevel cloud is the most frequent cloud type, and in the south subtropics low clouds are very frequent. However, similar to what is observed in the southeast of the Pacific and of the Atlantic Ocean, there is a region of high-level cloud systems starting from the African continent toward the southeast.

The contrast found in the low cloud cover between the northern and southern subtropics is in agreement with previous observations obtained using INSAT, ISCCP, and/or surface observations [Bony et al., 2000; Norris, 1998; Hahn et al., 1996]. In the northern subtropics, clear sky and small cumulus occur more than $90 \%$ of the time. Over the Arabian Sea the clear-sky frequency increases from January to March. This is also observed in the 1984-1994 ISCCP data and in the 19861989 INSAT studied by Bony et al. [2000]. Near the coast of India this frequency is greater than $80 \%$. In the eastern part of the south subtropics, low cloud occurrence frequency reach $80 \%$. This percentage decreases along the western side of the ocean and low cloud breakup.

However, in the southeastern subtropics, in the northern part of this region at the boundary with the ITCZ, as well as in the 1984-1994 ISCCP data and the 1986-1989 INSAT data, the clear-sky occurrence frequency is often greater than $40 \%$. This is not the case in 1999 for METEOSAT data analyzed with DCM. In this region, COF is less than $10 \%$.

In the ITCZ, for the 3-month period and the two data sets, the high cloud occurrence frequency is greater than $30 \%$ in a region going from Indonesia to North Madagascar. Near Indonesia this percentage is often greater than 55\%. In the ITCZ in February 1999 the high-level cloud cover frequency is less than in January and March. This is not the case in the ISCCP data set. From January to March, in the average over the 1984-1994 period, a decrease in high-level cloud cover and an increase of clear-sky occurrence frequency are observed. The DCM results seem to show that the 1999 year belongs to the years for which HOF is maximum near Indonesia.

The occurrence frequency of clear-sky pixels including very small fraction of low cloud (CL_BLOF) is in the average of $12 \%$. This percentage increases by a few percent in the regions with a high percentage of clear sky. The amount of pixels in the CL_BL class gives insight on the variation in COF which could be found with a threshold-based algorithm when using a threshold setup to avoid the inclusion of false clouds in the cloud cover or at the opposite a threshold setup to avoid the 
inclusion of pixels with very small clouds inside in the clear-sky pixel class.

This paper points out the complexity of making the analysis of interannual variations of cloud cover obtained with various cloud cover retrievals applied to different satellite data sets. Analyses of METEOSAT-7 data for the same 1999 period or, as soon as they will be available, the 1999 ISCCP D1 data will allow a more comprehensive interpretation of the differences observed in this study between 1999 and the previous years. To estimate uncertainties owing to the cloud retrieval itself, a comparison is undertaken with results provided by a threshold technique using the IR and WV radiances [Roca et al., this issue]. A study of the evolution of the cloud cover from morning to afternoon is also in progress.

Acknowledgments. Part of this work has been done during the stay of Hanna Pawlowska in the Laboratoire de Météorologie Dynamique in Paris (France). We acknowledge EUMETSAT which move METEOSAT -5 and provided the data freely. The ISCCP D1 data were obtained from NASA Langley Research Center EOSDIS Distributed Active Archive Center.

\section{References}

Ackerman, A., O. B. Toon, D. E. Stevens, A. Heymsfield, V. Ramanathan, and E. J. Welton, Reduction of tropical cloudiness by soot, Science, 288, 1042-1047, 2000.

Bony, S., W. D. Collins, and D. W. Fillmore, Indian Ocean low clouds during winter monsoon, J. Clim., 13, 2028-2043, 2000.

Brisson, A., P. Le Borgne, and A. Marsouin, Operational surface solar irradiance using METEOSAT data routine calibration and validation results, paper presented at Meteorological Satellite Data User's Conference: Geostationary Systems, Eur. Org. for the Exploit. of Meteorol. Satell., Vienna, Austria, Sept. 16-20, 1996.

Desbois, M., G. Sèze, and G. Szejwach, Automatic classification of clouds on Meteosat imagery: Application to high level clouds, J. Appl. Meteorol., 21, 401-412, 1982.

Hahn, C. J., S. G. Warren, and J. London, Edited synoptic cloud reports from ships and land stations over the globe, 1982-1991, Rep. ORNL/CDIAC77, NDP026B, Carbon Dioxide Inf. Anal. Cent., Oak Ridge Natl. Lab., Oak Ridge, Tenn., 1996.

Le Borgne, $P$., and A. Marsouin, Calibration of METEOSAT visible channels with desert targets and MODTRAN3.5 simulations, internal report, Cent. de la Meteorol. Spatiale, Meteo-France, Lannion, France, 1996.

Leon, J. F., et al., Large-scale advection of continental aerosols during INDOEX, J. Geophys. Res., this issue.

Minnis, P., K.-N. Liou, and Y. Takano, Inference of cirrus cloud properties using satellite-observed visible and infrared radiances, part I, Parameterization of radiances fields, J. Atmos. Sci., 50, 1279 1304, 1993.

Norris, J. R., Low cloud type over the ocean from surface observations, part II, Geographical and seasonal variations, J. Clim., 11, 383-403, 1998.

Platt, C. M. R., On the bispectral method for cloud parameter determination from satellite VISSR data: Separating broken cloud and S emitransparent cloud, J. Clim. Appl. Meteorol., 429-440, 1983.

Raffaelli, J. L., and G. Sèze, Cloud type separation using local correlation between visible and infrared satellite images, paper presented at Passive Infrared Remote Sensing of Clouds and the Atmosphere, SPIE-The Int. Soc. for Opt. Eng., Paris, Sept., 1995.

Ramanathan, V., et al., Indian Ocean Experiment (INDOEX): A multi-agency proposal for a field experiment in the Indian Ocean, C4 Publ. 162, Scripps Inst. of Oceanogr., La Jolla, Calif., June 1996.

Roca, R., and V. Ramanathan, Scale dependence of monsoonal convective systems over the Indian Ocean, J. Clim., 13, 1286-1298, 2000.

Roca, R., M. Viollier, L. Picon, and M. Desbois, A multisatellite analysis of deep convection and its moist environment over the Indian Ocean during the winter monsoon, J. Geophys. Res., this issue.

Rossow, W. B., and L. C. Garder, Validation of ISCCP cloud detections, J. Clim., 12, 2370-2393, 1993.

Rossow, W. B., and R. A. Schiffer, Advances in understanding clouds from ISCCP, Bull. Am. Meteorol. Soc., 80, 2261-2287, 1999.

Rossow, W. B., A. Walker, D. E. Beuschel, and M. D. Roiter, International Satellite Cloud Climatology Project (ISCCP) documentation of new cloud data sets, WMO/TD 737, 115 pp., World Meteorol. Org., Geneva, 1996.

Sèze, G., and M. Desbois, Cloud cover analysis from satellite imagery using spatial and temporal characteristics of the data, J. Clim. Appl. Meteorol., 26, 287-303, 1987.

Sèze, G., and W. B. Rossow, Time cumulated visible and infrared radiance histograms used as descriptor of surface and cloud variations, Int. J. Remote Sens., 12, 877-920, 1991.

Sèze, G., C. Vanbauce, J. C. Buriez, F. Parol, and P. Couvert, Cloud cover observed simultaneously from POLDER and METEOSAT, Phys. Chem. Earth, 24, 921-926, 1999.

Stubenrauch, C. J., W. B. Rossow, F. Chéruy, A. Chédin, and N. A. Scott, Clouds as seen by satellite sounders (3I) and imagers (ISCCP), part I, Evaluation of cloud parameters, J. Clim., 12, 21892213, 1999.

Twomey, S. A., Pollution and the planetary albedo, Atmos. Environ., 8, 1251-1256, 1974.

H. Pawlowska, Institute of Geophysics, Warsaw University, ul. Pasteura 7, 02-093 Warsaw, Poland. (hanna.pawlowska@igf.fuw.edu.pl)

G. Sèze, Laboratoire de Météorologie Dynamique du CNRS, Universite Pierre et Marie Curie, CNRS/UPMC-Boite 99, 75252 Paris Cedex 05, France. (genevieve.seze@lmd.jussieu.fr)

(Received October 10, 2000; revised February 14, 2001; accepted February 22, 2001.) 